
\title{
25 Research Square \\ PCDH9 target RAC1/ROS-NADPH-oxidase complex with the assistance of Pyk2 interaction through Cyclin D1 trafficking
}

Jiaojiao Zhang

Southern Medical University Fourth School of Clinical Medicine: Shenzhen Hospital of Southern Medical University of Marche

Hui-Zhi Yang

Dermatology Hospital of Southern Medical University;The Third Affiliated Hospital of Guangzhou Medical University

Shuang Liu

Dermatology Hospital of Southern Medical University

Md Obaidul Islam

Department of Clinical Sciences, Faculty of Medicine, Polytechnic University of Marche

\section{Yue Zhu}

College of Pharmaceutical Sciences, Guizhou University of Traditional Chinese Medicine;Nano-drug Technology Research Center at Guizhou University of Traditional Chinese Medicine

\section{RongYi Chen}

Dermatology Hospital of Southern Medical University

Zuhua Wang ( $\sim$ wangrui551601@163.com )

Guizhou University of Traditional Chinese Medicine https://orcid.org/0000-0001-7076-1014

\section{Research}

Keywords: PCDH9, RAC1, NADPH-oxidase, malignant melanoma, ROS, Pyk2, MMPs, MMP2, MMP9, Cyclin D1, protein-protein interaction (PPI).

Posted Date: October 21st, 2020

DOI: https://doi.org/10.21203/rs.3.rs-95026/v1

License: (c) (1) This work is licensed under a Creative Commons Attribution 4.0 International License. Read Full License 


\section{Abstract}

Background: Melanoma has dramatically increased during last 30 years with low five-year survival and prognosis rate.

Methods: Melanoma cells (A375 and G361) were chosen as in vitro model. The interference and overexpression of PCDH9 were infected by lentivirus. The effects of PCDH9 on melanoma cells were assessed in terms of alteration of PCDH9 such as cell viability, apoptosis, cell cycle and wound-healing assay. Moreover, expressions of PCDH9 with other genes (MMP2, MMP9, Pyk2 Cyclin D1, RAC1 and FAK) were also assessed by PCR and western blotting, immunohistochemical analysis. The protein complex immunoprecipitation was operated for protein-protein interaction (PPI) of PCDH9. Originlab 2020 was used for statistical analysis.

Results: Melanoma cells treated with PCDH9 showed influence melanoma as similar function as Hace1 that regulate RAC1/ROS-dependent NADPH oxidase complexes through targeting complex-bound RAC1 with the help of Pyk2, while RAC1 directly influence RAC1/ROS-NADPH-oxidase complex to induce metalloproteinases (MMPs), that are involved in cell migration and proliferation. Moreover, PCDH9 could influence the expressions of MMP2, MMP9, Pyk2, Cyclin D1 and RAC1, except FAK, decreased cell viability, cell migration and increased apoptosis, but did not exert significant effects on cell cycle. Besides, the immunohistochemical results exhibited lower PCDH9 expression in malignant melanoma samples than benign naevus tissue or/and normal skin, and PCDH9 was expressed in the cytoplasm rather than nuclei. The results of immunoprecipitation demonstrated that PCDH9 bind with RAC1 and Pyk2, suggesting the regulation of PCDH9 to melanoma progression.

Conclusion: Our results suggest that PCDH9 and RAC1 could predict for potential biomarkers prognosis of malignant melanoma, interestingly, with Pyk2's assistance like a bridge PCDH9 can modulate melanoma progression by interacting with RAC1.

\section{Background}

Cutaneous melanoma, a type of skin cancer, develops in melanocytes. According to data from the National Cancer Institute, the incidence of melanoma has rocketed over the last 30 years, even the Cancer Research UK estimated that melanoma has an incidence rate of 3.1/10000 and a mortality rate of $0.8 / 10000$ worldwide. With lower five-year survival rate of metastatic melanoma (MM), the prediction appeared essential. Albeit numbers of validated biomarkers have been confirmed in melanoma, Chinese population has been demonstrated to gain different subtypes of melanoma from the Caucasian population [1], therefore the already-being biomarkers haven't predicted well in prognosis of Chinese melanoma patients. Hence, a novel biomarker, feasible is urgently needed.

In order to figure out which genes could be relevant to Chinese population, bioinformatic technologies have been applied that PCDH9 have been chosen, while RAC1 always play opposite role was found in our natural bioproduct against melanoma study [2, 3]. PCDH9 belongs to protocadherins, one of the largest 
subfamilies of calcium-dependent adhesion proteins [4]. Previous studies have demonstrated that the overexpression of PCDH9 could suppress different cancers $[5,6]$ and tumor cells by arresting cell cycle at G0/G1 phase [7, 8], it also can predict prognosis of MM patients [5, 9]. Oppositely, RAC1 has been believed to affect actin cytoskeleton and regulate cell adhesion, motility, cycle, apoptosis, membrane trafficking and even transcription [10]. Both of them play important roles in tumor biology by modulating cell processes [11, 12]. Furtherly, activities of RAC1 have been reported of involving different stages of oncogenesis such as initiation, progression, invasion and metastasis [13], even it was ranked as the third most frequently occurring mutation in melanoma induced by UV $[14,15]$. Additionally, some reported ROS's involving in tumor cell migration and invasion $[16,17]$ and a key component of NAPDH-oxidasecomplex formed by RAC1, one of the major enzymatic sources of ROS in various tissues [18]. While it's reported that ROS/RAC1-NADPH-oxidase complex are involved in endothelial migration by mediation of angiotensin-1 (Ang-1) and vascular endothelial growth factor (VEGF) [19, 20]. As known, the endothelial migration is essential for tumor cell invasion, where ROS/RAC1-NADPH-oxidase complex induce expression of matrix metalloproteinases (MMPs) after growth factor and tumor promoter stimulation [21, 22]. Tumor-associated MMPs family has been studied for over half a century and are known as mediators in some cell processes [23]. Among them MMP2 has been believed to act as a pro-tumorigenic and pro-metastatic factor in different cancers besides melanoma [24], while higher MMP9 levels have been found in melanoma patients than healthy population that made it to be considered as a promising marker for melanoma [25]. Additionally, previous studies have found that MMP9 is activated during invasion of melanoma cells $[25,26]$. Therefore, we have chosen MMP2 and MMP9 as tumor metastasis indicators to explore the mechanism of how PCDH9 and RAC1 act on melanoma cells.

Proline-rich tyrosine kinase 2 (Pyk2) is a paralogue to focal adhesion kinase (FAK) in vertebrates [27] and, in absence of FAK, can execute the same therapeutic effects [28-30]. Increasing Pyk2 and FAK expression could trigger neoplastic activation and promote invasiveness with metastasis of different cancers, highlighting that Pyk2 is a potential therapeutic target for cancer [31, 32]. Some target molecules can influence, either increasing or decreasing, the function of Pyk2 by interaction [30]. Another key molecule of melanoma is the cell cycle regulator Cyclin D1 that has been demonstrated as an oncogene in cutaneous melanoma [33], and it could affect tumorigenesis via nuclear trafficking by multiple mechanisms [34].

Therefore, the main objective of this investigation was to clarify the role of PCDH9 and RAC1 in melanoma. After proceeding overexpression and interference of PCDH9 by GV358-PCDH9 lentivirus and GV358-SiRNA lentivirus in melanoma cell lines (A375 and G361), certain assays (cell viability, apoptosis and cell cycle assays, PCR, western blot analysis) and evaluation of PCDH9 expression by immunohistochemical (IHC) [35] in patients' skin biopsy were performed to explore the alterationinfluence of PCDH9 to melanoma cells.

\section{Results And Discussion}

\subsection{PCDH9 expression affected selected genes expression and proteins expression}


PCDH9 was overexpressed by lentivirus with PCDH9 plasmid (Fig. 1A) and interfered by lentivirus with siRNA (Fig. 1D, E). The relative expression of selected genes (Cyclin D1, Pyk2, MMP2 MMP9, RAC1, except FAK) varied with PCDH9 expression, but the effectiveness on them was different. PCDH9, Pyk2 and Cyclin D1 exhibited positive correlation (Fig. 1B, C, D, E), while RAC1, MMP2 and MMP9 exhibited negative in both melanoma A375 and G361 cells (Fig. 1B, C, D, E). The results of PCR were also confirmed by western blotting analysis. Except FAK, the protein expressions of Cyclin D1, Pyk2, MMP2 MMP9 and RAC1 had significant correlation with PCDH9 in G361 cells (Fig. 2B, D). Particularly, PCDH9, Pyk2 and Cyclin D1 exhibited positive correlation, while PCDH9 and RAC1, MMP2 and MMP9 exhibited negative correlation (Fig. 2). PCDH9 and selected protein expressions (Cyclin D1, Pyk2, MMP2 MMP9, RAC1) revealed the same tendency, but the effectiveness in A375 of PCDH9 overexpression was not significant $(p>0.05)$ (Fig. 2A). Therefore, overexpressed PCDH9 upregulated the gene expressions of Pyk2 and Cyclin D1 and downregulated RAC1, MMP1 and MMP9, while interfered PCDH9 downregulated protein expressions of Pyk2 and Cyclin D1 and upregulated RAC1, MMP1 and MMP9.

The expression of MMP2 is a prognostic marker for MM patients that could independently predict patient survival [36], its expression has been related to the poorer survival of melanoma patients [36], and the varieties of PCDH9 expression in melanoma cells were in accordance with this result. MMP9 could block the EGFR-ERK/AKT signaling pathway to suppress MM's angiogenesis and it has been considered as a key angiogenic factor [37]. The results of MMP9 expression in this study exhibited a negative correlation with PCDH9, as well as a positive correlation with RAC1. According to previous studies the role of Cyclin D1 in melanoma cells is debatable. Some studies suggested that the upregulated expression of Cyclin D1 enhances metastases [38], while others found a downregulation of Cyclin D1 in metastatic tumor and upregulation in tumor progression [39]. Even some histopathological studies showed that there is no significant difference in the expression of Cyclin D1 [40]. In the present study, we found that the expression of Cyclin D1 increased along with the overexpression of PCDH9, which is negatively correlated with MMP2 and MMP9.

\subsection{Effects of overexpressed PCDH9 on cell viability and apoptosis}

The overexpression of PCDH9 reduced the proliferation of melanoma cells. Overexpressed PCDH9 groups showed indeed a lower viability than control groups (Fig. 3A, B). As time passed, the viability of melanoma cells trended to stabilize, but PCDH9 overexpressed groups had less viable cells than control groups in different durations (Fig. 3C, D), and the differences between PCDH9 and control groups were significant (24, 48, 72 and 96 hours) (Fig. 3A, B).

The expression of PCDH9 significantly affected apoptosis of melanoma cells as well. Overexpression increased apoptosis $(p<0.05)$ (Fig. 3E), while interference decreased apoptosis $(p<0.05)$ in A375, a similar phenomenon happened in G361 with more modest effects (Fig. 3F).

Recent studies have found low PCDH9 expression in various cancer types [9, 41, 42]. The above phenomenon agreed with our investigation where alterations of PCDH9 could influence the viability of melanoma cells. Meanwhile, the percentage of apoptosis cells was affected by the change in PCDH9. 


\subsection{Effects of overexpressed PCDH9 on cell cycle and wound healing}

Regarding the cell cycle arrest, although we found differences between control and interfered PCDH9 groups in G361 cells, there was no discrepancy between overexpressed PCDH9 or interfered PCDH9 groups and other groups (blank and control groups) in both cell lines (A375 and G361) (Fig. 4A-D). Therefore, the changes in PCDH9 did not affect melanoma cell regulation and the cell cycle regulator Cyclin D1 modulation revealed no significant effect on cell cycle. Previous investigations of hepatocellular carcinoma (HCC) found that PCDH9 suppresses HCC cells by inducing cell cycle arrest at G0/G1 phase [7]. This result suggests that PCDH9 and Cyclin D1 could affect melanoma cell through other mechanisms.

With respect to cell migration, after quantifying the scratched boundary by Image J (Fig. 4E, F), the results revealed that the relative density decreased with the duration of cell culture in blank and control groups $(p<0.001)$, while the relative wound density did not change in a marked way in overexpressed PCDH9 groups $(p>0.05)$ (Fig. 4G, H). The relative wound density of scratched boundary was significantly different in overexpressed PCDH9 groups compared with blank and control groups after 24 hours and 48 hours $(p<0.001)$ (Fig. $4 \mathrm{l}, \mathrm{J})$. This phenomenon is similar to the correlation found between PCDH9 and other tumor cells (HCC) $[42,43]$. To conclude, overexpressed PCDH9 could significantly inhibit melanoma cell migration, by decreasing cell viability and increasing apoptosis.

\subsection{Interaction of PCDH9 with RAC1 and Pyk2}

The interaction between Pyk2 and other proteins, as well as targeted molecules, could affect the function of Pyk2 by interaction as previously demonstrated $[2,38]$. In the current study, expression of Pyk2 in melanoma cells was significantly affected by the alteration of PCDH9 (Fig. 1B, C) (Fig. 2B). Therefore, we explored the interaction between "PCDH9 and Pyk2", "PCDH9 and RAC1" by Co-IP. "PCDH9 and Pyk2", "PCDH9 and RAC1" were co-immunoprecipitated with PCDH9-FLAG in both cell lines (A375-PCDH9-Flag and G361-PCDH9-Flag), while there was no protein in negative control (Supplementary Figure S1). It was demonstrated that PCDH9 interacted with the protein Pyk2 and the protein RAC1. We assumed in accordance with previous investigations that PCDH9 may serve the function as Hace1 that regulate RAC1/ROS-dependent NADPH oxidase complexes through similar mechanism [44], but Hace1 target complex-bound RAC1 directly, while PCDH9 need the help of Pyk2 [44].

\subsection{PCDH9 protein expressed differences in normal skin, pigmented nevus and melanoma tissue tested by IHC stains}

Immunohistochemical results showed that the positive percentage of PCDH9 expression was lower in human melanoma tissue than in normal skin or/and pigmented nevus tissue; additionally, PCDH9 was mainly expressed in the cytoplasm, while a small amount was expressed in the nuclei. The positive percentage of PCDH9 was expressed in normal skin or/ and pigmented nevus tissue, while only $23.3 \%$ (7/30) in melanoma tissue, which was lower than non-tumor tissue (Table 1, Fig. 5). 
Table 1

The positive percentage of PCDH9 expression in normal skin, pigmented nevus, and melanoma tissues.

\begin{tabular}{|lllll|}
\hline Time (hours) & Total & PCDH9(-) & PCDH9(+) & Positive Percentage \\
\hline Normal skin & 45 & 0 & 45 & $100.0 \%$ \\
\hline $\begin{array}{l}\text { Pigmented } \\
\text { nevus }\end{array}$ & 30 & 0 & 30 & $100.0 \%$ \\
\hline Melanoma & 30 & 23 & 7 & $23.3 \%$ \\
\hline
\end{tabular}

Previous investigation exhibited that Hace1 target complex-bound RAC1 to regulate Cyclin D1 expression, ROS generation, and high Cyclin D1 express with ROS-induced DNA damage [44]. High DNA damage susceptibility tend to Carcinogenesis. Meanwhile, previous exploration of how Cyclin D1 affects tumorigenesis speculated that it could play a role in nuclear trafficking rather than overexpressing itself $[34,44]$. PCDH9 might be trafficked from nuclei to cytoplasm by the assistance of Cyclin D1.

\section{Conclusion}

According to previous studies, MMP2 and MMP9 could represent a biomarker of malignant melanoma $[24,25]$, while Pyk2 and FAK expression could trigger neoplastic activation and promote invasiveness with metastasis of different cancers [31,32]. We explored and performed a series of investigations, including cell viability assay, apoptosis assay, PCR, Co-IP, of interference and overexpression of PCDH9 by GV358-PCDH9 lentivirus and GV358-SiRNA lentivirus respectively in melanoma A375 and G361 cell lines. According to our results, overexpressed PCDH9 triggered upregulated expressions of Pyk2 and Cyclin D1, while RAC1, MMP1 and MMP9 were downregulated, and apoptosis of melanoma cell lines was promoted. Interfered PCDH9 induced downregulation of Pyk2 and Cyclin D1, while RAC1, MMP1 and MMP9 were upregulated and decreased apoptosis. However, the alteration of PCDH9 expression did not affect melanoma cell regulation in a significant manner. It has been suggested that PCDH9 could affect melanoma cells by different mechanisms. Specimen investigations of IHC assay exhibited lower PCDH9 expression in malignant melanoma specimens than in benign naervus tissue or/and normal skin, furtherly proving the indicative function of PCDH9; our study revealed that PCDH9 was mainly expressed in the cytoplasm rather than in nuclei. The trafficking of PCDH9 might be through Cyclin D1, and Cyclin D1 could play a more important role in trafficking than in cell regulation. Co-IP demonstrated the interaction between "PCDH9 and Pyk2", "PCDH9 and RAC1". The function of Pyk2 can be modulated by interaction with target molecules [30], PCDH9 could affect Pyk2 by interacting with it. Though PCDH9 and RAC1 showed opposite phenomena, they can interact with each other. PCDH9 and RAC1 not only could predict the prognosis of malignant melanoma, but also modulate melanoma progression. It is that increase ROS generation and enhance NADPH oxidase activity complex result in hyperactive angiogenesis. PCDH9 may serve the function as Hace1 that regulate RAC1/ROS-dependent NADPH 
oxidase complexes through similar mechanism [44], but Hace1 target complex-bound RAC1 directly, while PCDH9 need the help of Pyk2 [44]. Meanwhile, when Hace1 interact with RAC1, it may cause lower Cyclin D1 expression; but our study here, Cyclin1 might act as a transporter of PCDH9.

\section{Methods}

\subsection{Chemical and Antibodies}

Ethanol, isopropyl alcohol, Triton X-100 were obtained from Sigma chemicals (Shanghai, China), DMEM, Fetal Bovine Serum, DEPC-treated water (Ambion, Inc.), TRIzol reagent (invitrogen), BCA protein assay kit were purchased from Thermo Fisher Scientific (Shanghai, China); immobilon PVDF membranes, immobilon western chemiluminescent substrates were bought from Merck Millipore (Darmstadt, Germany); water was obtained from EPED-20TF (Nanjing, China), Cell Counting Kit-8 (CCK-8) were purchased from Dongren Chemical Technology (Shanghai, China); primers (PCDH9, RAC1, Cyclin D1, Pyk2, FAK, MMP2, MMP9 and GAPDH) was designed and cDNA synthesized by Sangon Biotech (Shanghai, China); GV358-PCDH9 lentivirus, GV358-SiRNA lentivirus were designed by Genechem (Shanghai, China); SYBR ${ }^{\circledR}$ Premix Ex Taq $^{\text {TM }}$ Ex Taq $^{\text {TM }}$ II and PrimeScript ${ }^{\text {TM }}$ RT reagent Kit with gDNA Eraser were bought from Takara Bio Inc. (Beijing, China); primary antibodies (Cyclin D1, Pyk2, FAK, MMP2, MMP9, and RAC 1, beta-tubulin), secondary goat anti-rabbit immunoglobulin G-Peroxidase antibody protease/phosphatase inhibitor were purchased from Cell Signaling Technology (Shanghai, China); primary antibodies (PCDH9) and protease inhibitor cocktail was purchased from abcam (Shanghai, China).

\subsection{Cell culture}

Both cell lines A375 and G361 (ATCC $\AA$ CRL-1619 ${ }^{\mathrm{TM}}$ ) were bought from the American Type Culture Collection (ATCC) (MD, USA). They were grown in Dulbecco's Modified Eagle's Medium (DMEM) supplemented with $10 \%$ heat-inactivated fetal bovine serum as well as $100 \mathrm{IU} / \mathrm{mL}$ penicillin and $100 \mu \mathrm{g} / \mathrm{mL}$ streptomycin. Cells were maintained in a $\mathrm{CO}_{2}$ incubator at $37^{\circ} \mathrm{C}$ under a humidified atmosphere (95\% air, 5\% CO2).

\subsection{Sample collection and Preparation}

Tissues (human normal skin tissue $(n=45)$, human pigmented nevus $(n=30)$ and primary malignant melanoma tissue $(n=30)$ ) were collected and prepared as paraffin specimens until use. These tissues were ethically acquired from the outpatient clinic of the Affiliated Hospital of Guangdong Medical University with Chinese population (Han people) with personal identifiers redacted.

\subsection{Transfection}

Melanoma cells (A375 and G361) were seeded in 6 well plates $\left(1 \times 10^{5}\right.$ cell/well) the day before transfection and were transfected by two types of lentiviruses (siRNA and PCDH9). Control group were transfected with the empty vector. Blank group were treated with transfection reagent only. Transfection was performed using Genechem Transfection Reagent (Shanghai, China), according to the 
manufacturer's instructions. Seventy-two hours after transfection, cells were observed by fluorescent inverted microscope for being screened by puromycin. The efficiency of PCDH9 alteration in melanoma cells was detected by Real-Time PCR, Western blot analysis.

\subsection{Cell viability by Cell Counting Kit-8 (CCK-8)}

Cells were seeded into 96 -well plates at a density of $2 \times 10^{5}$ cells/well and treated by non-transfected plasmid, transfected with empty plasmid and transfected with PCDH9 overexpressed plasmid as explained above. After incubation at $24 \mathrm{~h}, 48 \mathrm{~h}, 72 \mathrm{~h}$ and $96 \mathrm{~h}, 10 \mu \mathrm{L}$ of CCK-8 were added to each well, and cells were incubated for another $4 \mathrm{~h}$ at $37^{\circ} \mathrm{C}$. The level of colored formazan derivative was analyzed on Thermo Scientific Multiskan FC (Vantaa, Finland) at a wavelength of $450 \mathrm{~nm}$ (Ishiyama, Miyazono, Sasamoto, Ohkura, \& Ueno, 1997; Tominaga et al., 1999). The viable cells were directly proportional to the formazan production and the percentage of viable ones was calculated. The following equations (Eq. 1 and Eq. 2) were utilized to determine the viability rate and inhibition rate respectively.

\section{$\mathrm{V} \%=\frac{\mathrm{As}-\mathrm{Ab}}{\mathrm{Ac}-\mathrm{Ab}} \times 100 \% \quad$ (Equation 1) \\ $\mathrm{I} \%=\frac{\mathrm{Ac}-\mathrm{As}}{\mathrm{Ac}-\mathrm{Ab}} \times 100 \% \quad$ (Equation 2)}

V\%: the viability rate

$A_{s}$ : the absorbing values of experimental wells (cells with medium, CCK-8, PCDH9 overexpressed plasmid)

$A_{b}$ : the absorbing values of blank wells (medium, CCK-8, empty plasmid)

$A_{c}$ : the absorbing values of control wells (cells with medium, CCK-8)

I\%: the inhibition rate

\subsection{Apoptosis analysis}

Apoptosis was analyzed by cytometric analysis, using FITC Annexin V Apoptosis Detection Kit (company name). Cells were seeded in 6-well plate at a density of $1 \times 10^{6}$ cells/well. Briefly, cells were treated with Camptohtecin stock solution ( $1 \mu \mathrm{L}$ in DMSO) and incubated for 5 hours at $37^{\circ} \mathrm{C}$. After that, the cells were centrifuged (1000 rpm for $10 \mathrm{~min}$ ), washed twice with cold PBS and resuspended in 1X Binding $5 \mu \mathrm{L}$ of FITC Annexin V and $5 \mu \mathrm{L} \mathrm{PI} \mathrm{(Bio-Rad)} \mathrm{were} \mathrm{added} \mathrm{to} \mathrm{cell} \mathrm{suspension} \mathrm{and} \mathrm{incubated,} \mathrm{protected} \mathrm{from} \mathrm{light,}$ for $15 \mathrm{~min}$ at room temperature. Finally, samples were analyzed using the BD FACS Canto II flow cytometer.

\subsection{Cell cycle assay}


Cell cycle was analyzed using the flow apoptosis. Briefly, Cells were seeded in 6-well plates at a density of $1 \times 10^{6}$ cells/well. Cells were then detached, centrifuged at (1000 rpm for $10 \mathrm{~min}$ ) and then vortexed with 5 $\mathrm{mL}$ cold $75 \%$ ethanol. Cells were incubated at $-20^{\circ} \mathrm{C}$ for 2 hours, washed twice with PBS to remove ethanol. Cells were resuspended in $0.5 \mathrm{~mL} \mathrm{PI/RNase} \mathrm{staining} \mathrm{Buffer} \mathrm{(Bio-Rad)} \mathrm{for} 15 \mathrm{~min}$ at room temperature, samples were analyzed using the BD FACS Canto II flow cytometer.

\subsection{Wound-healing assay}

Cells were seeded into 6 -well plates at a density of $1.5 \times 105$ cells/well until reach on confluency (80-100 $\%$ ) and scratched by a sterile $10 \mu \mathrm{L}$ pipette tip. Cells were washed twice with PBS; then complete medium was added to allow cells moving into the gap, photographed by using an inverted microscope DMI3000B (Leica, Germany) at 0h, 24h and 48h. The image J (Maryland, USA) was used to measure the wound space. Migration rate was calculated as the proportion of initial scratch distant of each sample and the mean distance between both borderline remaining cell free after migration.

\subsection{Quantitative Real-Time PCR analysis}

Each frozen pellet of melanoma cells (A375 and G361), treated in different experimental conditions were homogenized in a lysis buffer. Total RNA was isolated through the TRIzol Reagent Total RNA isolation system (Thermo Fisher Scientific, United States) according to the manufacturer's reference guide. Total RNA was quantified by nanodrop, was reverse transcribed by PrimeScript RT reagent kit TaKaRa referred to SYBR Green qPCR assay introduction (SYBR® Premix Ex TaqTM II kit) by MasterCycler Gradient PCR (Thermo Fisher Scientific, United States). The reaction mixture $(20 \mu \mathrm{L})$ was taken and incubated for three minutes at $95^{\circ} \mathrm{C}$. Quantification of genes was performed with the $2^{-\triangle \triangle C T}$ method, as described previously [45]: the sample was cycled $\left(95^{\circ} \mathrm{C}-10 \mathrm{sec}\right.$ and $\left.60^{\circ} \mathrm{C}-20 \mathrm{sec}\right)$ for 40 times by ABI7500Fast Real-time PCR System Amplifier (Thermo Fisher Scientific, United States). The primers designed for selected genes (PCDH9, GAPDH, Pyk2, Cyclin D1, MMP2, MMP9, RAC1 and FAK) and amplicon sizes are shown in Supplementary Table S1.

\subsection{Western Blot Analysis}

Cells were lysed in a buffer containing $20 \mathrm{mM}$ Tris $-\mathrm{HCl}(\mathrm{pH}=7.5), 0.9 \% \mathrm{NaCl}, 0.2 \%$ Triton $\mathrm{X}-100$, and $1 \%$ of the protease inhibitor cocktail. Equal amounts of protein $30 \mu \mathrm{g}$ of each sample were subjected to $10 \%$ acrylamide sodium dodecyl sulfate polyacrylamide gel electrophoresis and transferred to immobilon PVDF membranes. Membranes were washed three times with PBS, blocked with $5 \%$ non-fat dry, and incubated with specific primary antibodies at $4^{\circ} \mathrm{C}$ overnights. Next, membranes were washed with TBST and incubated with secondary antibodies according to the manufacturer's instructions. Protein bands were visualized using Immobilon Western Chemiluminescent Substrate, and the protein signals were detected by Azure c500 Infrared Western Blot Imaging System from Azure Biosystems (CA, USA). Quantification of protein expression was made using the software Image $J$ (Maryland, USA) and Origin 2020 for visualization (Northampton, MA, USA). 


\subsection{Immunohistochemical (IHC) assay}

The paraffin specimens were deparaffinizated included two 100\% xylene changes (xylene $1-10$ min, xylen II-10 $\mathrm{min}$ ) followed by rehydration with a graded series of ethanol (anhydrous ethanol I-5min, anhydrous ethanol II-5 min, 95\% ethanol-5 $\mathrm{min}, 85 \%-5 \mathrm{~min}, 75 \%-5 \mathrm{~min}$ ) and then rinsed under distilled running water for 3-5 min. Antigen retrieval consisted of a two-minute incubation of slides in citric acid retrieval solution heated to $98^{\circ} \mathrm{C}$ with a commercial steamer following a cool down step to room temperature (cold water and ice pack were added), slides were transferred into a wet box and were then rinsed three times by PBS. After protein blocking, primary antibodies (1:200) were incubated at $4^{\circ} \mathrm{C}$ overnight. After being in room temperature for 30 min, the slides were washed 3 times for 3 min each by PBS. After removing PBS and protein blocking, secondary antibodies (1:1000) were added at room temperature for $1 \mathrm{~h}$. The slides were then washed 3 times for 3 min each by PBS. After removing PBS, 1 drop of prepared DAB solution ( $1 \mathrm{ml}$ $A: 1$ drop $B: 1$ drop $C$ ) for DAB staining was added and the slides were observed under microscope. After being rinsed in running water for $10 \mathrm{~min}$, hematoxylin was added for $1 \mathrm{~min}$ and then the slides were washed by water for $5 \mathrm{~min}$. The slides were then dehydrated in a series of ethanol (75\%, 85\%, 95\%, 100\%) and $100 \%$ xylene changes, and mounted with a coverslip with dry neutral resin.

\subsection{Evaluation of various protein expression in MM}

Evaluation of various protein expressions in MM were evaluated by semi-quantitative analysis, according to the staining intensity and the percentage of positive cells. The score standards of staining intensity were no coloration-0, low intensity (light yellow)-1, medium intensity (light brown)-2, and high intensity (dark brown)-3. Five fields of view were randomly selected under microscope (400x), and 500 cells were counted as one unit, meanwhile the percentage of positive cells was calculated. The percentage scores were as follows: $<5 \%-0,6 \%$ to $25 \%-1,26 \sim 50 \%-2 ; 51 \% \sim 75 \%-3,>75 \%-4$. The score standards were the product of staining intensity and percentage of positive cells: 0 -negative (-), 1 to 4 -positive (+), 5 to 8 moderately positive (++), and 9 to 12 - strongly positive (+++).

\subsection{Protein complex immunoprecipitation (Co-IP) assay}

PCDH9-Flag was overexpressed in A375 and G361 cell lines by Lentiviruses Infection and cultured by DMEM with $10 \%$ FBS in $10 \mathrm{~cm}$ petri dishes, as mentioned above. After discarding the cell media and washing once by iced PBS, the cells were lysed in a buffer containing TBS with $1 \%$ Triton X-100 and PMSF, $1 \mathrm{~mL}$ in each dish. The solution was collected in $1.5 \mathrm{~mL}$ Eppendorf tubes and after centrifugation (1000 rpm for $15 \mathrm{~min}$ ), the supernatant was collected. For the input sample preparation, $80 \mu \mathrm{L}$ of protein solution with $20 \mu \mathrm{L}$ ( $5 \times$ loading buffer) were boiled. The leftover solution was incubated with $2 \mu \mathrm{L}$ of antiFlag antibody at $4^{\circ} \mathrm{C}$ overnight. Protein $\mathrm{G}$ Agarose beads ( $40 \mu \mathrm{L}$ each tube) were added and the solution was shaken for $3 \mathrm{~h}$ at $4{ }^{\circ} \mathrm{C}$. Then after being centrifuged ( $2500 \mathrm{rpm}$ for $5 \mathrm{~min}$ ), the supernatant was removed. The sediments were washed five times by lysis buffer with PMSF ( $1 \mathrm{~mL}$ each time) and after the addition of loading buffer $(40 \mu \mathrm{L})$ were boiled for $10 \mathrm{~min}$ at $100^{\circ} \mathrm{C}$. Control group was incubated with mouse lgG. 


\subsection{Statistical Analysis}

Data are presented as the mean \pm SD from at least 3 independent experiments. Statistical comparisons between two groups were made using Student's $t$ tests. Differences among three or more groups were compared by one-way ANOVA followed by least significant difference post hoc tests (Originlab 2020, Northampton, MA, USA), $p<0.05$ was considered as statistically significant, while $p<0.01$ was considered as highly statistically significant.

\section{List Of Abbreviations}

Acral lentiginous melanoma (ALM); Angiotensin-1 (Ang-1); Cell Counting Kit-8 (CCK-8); Complex immunoprecipitation (Co-IP); Focal adhesion kinase (FAK); Hepatocellular carcinoma (HCC); Immunohistochemical (IHC); Malignant melanoma (MM); Metalloproteinases (MMPs); Proline-rich tyrosine kinase 2 (Pyk2); Protocadherin-9 (PCDH9); Ras-related C3 botulinum toxin substrate 1 (RAC1); Vascular endothelial growth factor (VEGF).

\section{Declarations}

Ethics approval and consent to participate: The experimental protocol was established, according to the ethical guidelines of the Helsinki Declaration and was approved by the Human Ethics Committee of the Affiliated Hospital of Guangdong Medical University. Written informed consent was obtained from individual or guardian participants.

Consent for publication: Availability of data and material.

Availability of data and materials: All data generated or analyzed during this study are included in this published article [and its supplementary information files.

Competing Interest: The authors declare that they have no competing interests.

Funding: This research was supported by Natural Science Foundation of Guangdong Province of China 2016 A030313682 and Natural Science Foundation of Guangdong Province of China 2020A1515010281.

Author Contributions: Conceptualization: JJ Zhang, RY Chen, ZH Wang. Data curation: JJ Zhang, S Liu. Formal Analysis: JJ Zhang, HZ Yang, Y Zhu, S Liu, MO Islam. Project Administration: RY Chen, ZH Wang. Resources: RY Chen. Visualization: JJ Zhang. Writing original draft: JJ Zhang. Review and Editing: JJ Zhang, RY Chen, MO Islam.

Acknowledgements: Thanks Maurizio Battino and Francesca Giampieri (Department of Clinical Sciences, Faculty of Medicine, Polytechnic University of Marche, Ancona, Italy), Gianluca Storci (Department of experimental, diagnostic and specialty medicine, University of Bologna, Italy) for their insight and helpful discussion. We also thank Dr. Jingquan He (Shanghai Biotree Biotechnology Ltd, Shanghai, China) for his company and his supports of Protein complex immunoprecipitation. 


\section{References}

1. Utikal J, Becker JC, Ugurel S: Diagnostic and prognostic biomarkers in melanoma: current state of play. In: Diagnostic and Prognostic Biomarkers and Therapeutic Targets in Melanoma. Springer; 2012: 9-18.

2. Chen R, Zhang G, Zhou Y, Li N, Lin J: A time course-dependent metastatic gene expression signature predicts outcome in human metastatic melanomas. Diagn Pathol 2014, 9(1):155.

3. Chen R, Fu M, Zhang G, Zhou Y, Zhu S, Liu J, Wang D, Deng A, Wang Z: Rac1 regulates skin tumors by regulation of keratin 17 through recruitment and interaction with $\mathrm{CD} 11 \mathrm{~b}+\mathrm{Gr}+\mathrm{cells}$. Oncotarget 2014, 5(12):4406.

4. Kim SY, Yasuda S, Tanaka H, Yamagata K, Kim H: Non-clustered protocadherin. Cell Adh Migr 2011, 5(2):97-105.

5. Wang C, Yu G, Liu J, Wang J, Zhang Y, Zhang X, Zhou Z, Huang Z: Downregulation of PCDH9 predicts prognosis for patients with glioma. J Clin Neurosci 2012, 19(4):541-545.

6. Bidoki SH, Bayatani A, Sarlak M, Rasouli M, Mostafaie P, Saghafi M, Negari AT: Assessing Expression of TGF-B2 and PCDH9 Genes in Breast Cancer Patients. Age 2018, 7:20.

7. Lv J, Zhu P, Zhang X, Zhang L, Chen X, Lu F, Yu Z, Liu S: PCDH9 acts as a tumor suppressor inducing tumor cell arrest at G0/G1 phase and is frequently methylated in hepatocellular carcinoma. $\mathrm{Mol} M e d$ Report 2017, 16(4):4475-4482.

8. Wang C, Tao B, Li S, Li B, Wang X, Hu G, Li W, Yu Y, Lu Y, Liu J: Characterizing the role of PCDH9 in the regulation of glioma cell apoptosis and invasion. J Mol Neurosci 2014, 52(2):250-260.

9. Chen Y, Xiang H, Zhang Y, Wang J, Yu G: Loss of PCDH9 is associated with the differentiation of tumor cells and metastasis and predicts poor survival in gastric cancer. Clin Exp Metastasis 2015, 32(5):417-428.

10. Espina C, Cespedes MV, Garcia-Cabezas MA, Gomez del Pulgar MT, Boluda A, Oroz LG, Benitah SA, Cejas $\mathrm{P}$, Nistal M, Mangues $\mathrm{R}$ et al: A critical role for Rac1 in tumor progression of human colorectal adenocarcinoma cells. Am J Pathol 2008, 172(1):156-166.

11. Aznar S, Fernandez-Valeron P, Espina C, Lacal JC: Rho GTPases: potential candidates for anticancer therapy. Cancer Lett 2004, 206(2):181-191.

12. del Pulgar TG, Benitah SA, Valerón PF, Espina C, Lacal JC: Rho GTPase expression in tumourigenesis: evidence for a significant link. Bioessays 2005, 27(6):602-613.

13. Vega FM, Ridley AJ: Rho GTPases in cancer cell biology. FEBS Lett 2008, 582(14):2093-2101.

14. Davis MJ, Ha BH, Holman EC, Halaban R, Schlessinger J, Boggon TJ: RAC1P29S is a spontaneously activating cancer-associated GTPase. Proc Natl Acad Sci U S A 2013, 110(3):912-917.

15. Ellenbroek SI, Collard JG: Rho GTPases: functions and association with cancer. Clin Exp Metastasis 2007, 24(8):657-672.

16. Bae YS, Oh H, Rhee SG, Yoo YD: Regulation of reactive oxygen species generation in cell signaling. Mol Cells 2011, 32(6):491-509. 
17. Wu WS: The signaling mechanism of ROS in tumor progression. Cancer Metastasis Rev 2006, 25(4):695-705.

18. Bedard K, Krause KH: The NOX family of ROS-generating NADPH oxidases: physiology and pathophysiology. Physiol Rev 2007, 87(1):245-313.

19. Harfouche R, Malak NA, Brandes RP, Karsan A, Irani K, Hussain SN: Roles of reactive oxygen species in angiopoietin-1/tie-2 receptor signaling. FASEB J 2005, 19(12):1728-1730.

20. Ushio-Fukai M, Alexander RW: Reactive oxygen species as mediators of angiogenesis signaling. Role of NAD (P) H oxidase. Mol Cell Biochem 2004, 264(1-2):85-97.

21. Binker MG, Binker-Cosen AA, Richards D, Oliver B, Cosen-Binker LI: EGF promotes invasion by PANC-1 cells through Rac1/ROS-dependent secretion and activation of MMP-2. Biochem Biophys Res Commun 2009, 379(2):445-450.

22. Steinbrenner H, Ramos MC, Stuhlmann D, Mitic D, Sies H, Brenneisen P: Tumor promoter TPA stimulates MMP-9 secretion from human keratinocytes by activation of superoxide-producing NADPH oxidase. Free Radic Res 2005, 39(3):245-253.

23. Overall CM, Lopez-Otin C: Strategies for MMP inhibition in cancer: innovations for the post-trial era. Nat Rev Cancer 2002, 2(9):657-672.

24. Marusak C, Bayles I, Ma J, Gooyit M, Gao M, Chang M, Bedogni B: The thiirane-based selective MT1MMP/MMP2 inhibitor ND-322 reduces melanoma tumor growth and delays metastatic dissemination. Pharmacol Res 2016, 113(Pt A):515-520.

25. Bianchini F, D'Alessio S, Fibbi G, Del Rosso M, Calorini L: Cytokine-dependent invasiveness in B16 murine melanoma cells: role of uPA system and MMP-9. Oncol Rep 2006, 15(3):709-714.

26. Tang ZY, Liu Y, Liu LX, Ding XY, Zhang H, Fang LQ: RNA i-mediated MMP-9 silencing inhibits mouse melanoma cell invasion and migration in vitro and in vivo. Cell Biol Int 2013, 37(8):849-854.

27. Corsi JM, Rouer E, Girault JA, Enslen H: Organization and post-transcriptional processing of focal adhesion kinase gene. BMC Genomics 2006, 7(1):198.

28. Sieg DJ, Ilic D, Jones KC, Damsky CH, Hunter T, Schlaepfer DD: Pyk2 and Src-family protein-tyrosine kinases compensate for the loss of FAK in fibronectin-stimulated signaling events but Pyk2 does not fully function to enhance FAK- cell migration. EMBO J 1998, 17(20):5933-5947.

29. Weis SM, Lim ST, Lutu-Fuga KM, Barnes LA, Chen XL, Gothert JR, Shen TL, Guan JL, Schlaepfer DD, Cheresh DA: Compensatory role for Pyk2 during angiogenesis in adult mice lacking endothelial cell FAK. J Cell Biol 2008, 181(1):43-50.

30. Naser R, Aldehaiman A, Díaz-Galicia E, Arold ST: Endogenous control mechanisms of FAK and PYK2 and their relevance to cancer development. Cancers (Basel) 2018, 10(6):196.

31. Yoon H, Dehart JP, Murphy JM, Lim ST: Understanding the roles of FAK in cancer: inhibitors, genetic models, and new insights. J Histochem Cytochem 2015, 63(2):114-128.

32. Zhu X, Bao Y, Guo Y, Yang W: Proline-Rich Protein Tyrosine Kinase 2 in Inflammation and Cancer. Cancers (Basel) 2018, 10(5):139. 
33. Sauter ER, Yeo U-C, von Stemm A, Zhu W, Litwin S, Tichansky DS, Pistritto G, Nesbit M, Pinkel D, Herlyn M: Cyclin D1 is a candidate oncogene in cutaneous melanoma. Cancer Res 2002, 62(11):3200-3206.

34. Kim JK, Diehl JA: Nuclear cyclin D1: an oncogenic driver in human cancer. J Cell Physiol 2009, 220(2):292-296.

35. Dinehart MS, Dinehart SM, Sukpraprut-Braaten S, High WA: Immunohistochemistry utilization in the diagnosis of melanoma. J Cutan Pathol 2020, 47(5):446-450.

36. Rotte A, Martinka M, Li G: MMP2 expression is a prognostic marker for primary melanoma patients. Cell Oncol 2012, 35(3):207-216.

37. Li L, Fan P, Chou H, Li J, Wang K, Li H: Herbacetin suppressed MMP9 mediated angiogenesis of malignant melanoma through blocking EGFR-ERKJAKT signaling pathway. Biochimie 2019, 162:198207.

38. Ramirez JA, Guitart J, Rao MS, Diaz LK: Cyclin D1 expression in melanocytic lesions of the skin. Ann Diagn Pathol 2005, 9(4):185-188.

39. Oba J, Nakahara T, Abe T, Hagihara A, Moroi Y, Furue M: Expression of c-Kit, p-ERK and cyclin D1 in malignant melanoma: an immunohistochemical study and analysis of prognostic value. $J$ Dermatol Sci 2011, 62(2):116-123.

40. George E, Polissar NL, Wick M: Immunohistochemical evaluation of p16INK4A, E-cadherin, and cyclin D1 expression in melanoma and Spitz tumors. Am J Clin Pathol 2010, 133(3):370-379.

41. Wang C, Chen Q, Li S, Li S, Zhao Z, Gao H, Wang X, Li B, Zhang W, Yuan Y et al: Dual inhibition of PCDH9 expression by miR-215-5p up-regulation in gliomas. Oncotarget 2017, 8(6):10287-10297.

42. Xie Z, Zhou F, Yang Y, Li L, Lei Y, Lin X, Li H, Pan X, Chen J, Wang G: Lnc-PCDH9-13: 1 is a hypersensitive and specific biomarker for early hepatocellular carcinoma. EBioMedicine 2018, 33:5767.

43. Zhu P, Lv J, Yang Z, Guo L, Zhang L, Li M, Han W, Chen X, Zhuang H, Lu F: Protocadherin 9 inhibits epithelial-mesenchymal transition and cell migration through activating GSK-3 $\beta$ in hepatocellular carcinoma. Biochem Biophys Res Commun 2014, 452(3):567-574.

44. Daugaard M, Nitsch R, Razaghi B, McDonald L, Jarrar A, Torrino S, Castillo-Lluva S, Rotblat B, Li L, Malliri A et al: Hace1 controls ROS generation of vertebrate Rac1-dependent NADPH oxidase complexes. Nat Commun 2013, 4(1):2180.

45. Livak KJ, Schmittgen TD: Analysis of relative gene expression data using real-time quantitative PCR and the 2- $\Delta$ ACT method. Methods 2001, 25(4):402-408.

\section{Figures}



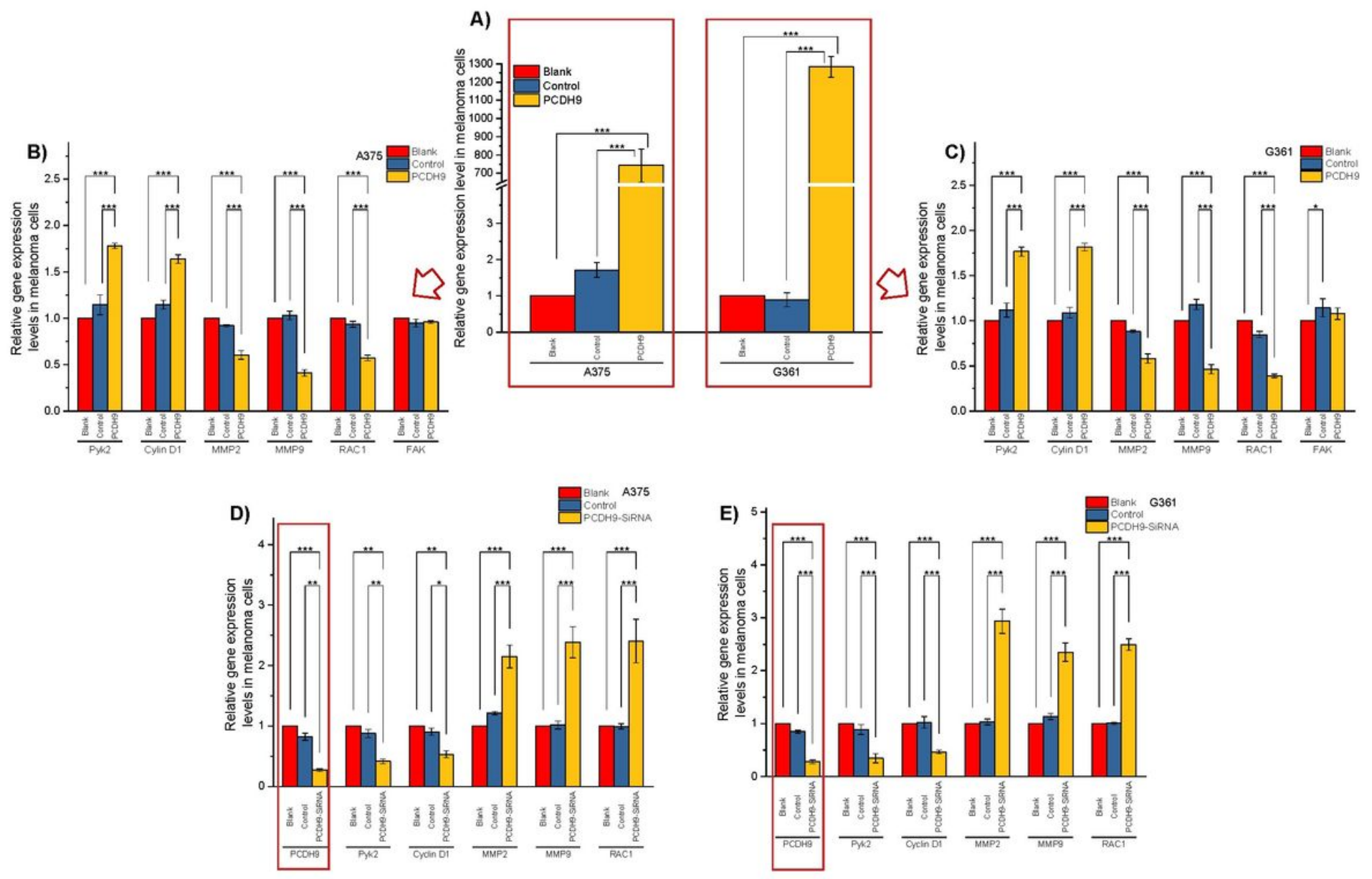

\section{Figure 1}

Effects of overexpressed and interfered PCDH9 in melanoma cells measured by PCR analysis. (A) The expressions of $\mathrm{PCDH} 9$ were significantly upregulated by lentivirus infection. Overexpressed PCDH9 significantly upregulated the gene expressions of Pyk2 and Cyclin D1 and downregulated RAC1, MMP1 and MMP9 except FAK in A375 (B) and G361 (C) cells. Interfered PCDH9 downregulated the gene expressions of Pyk2 and Cyclin D1 and upregulated RAC1, MMP1 and MMP9 in A375 (D) and G361 (E) cells. ${ }^{*} p<0.05,{ }^{*} p<0.01,{ }^{\star \star \star} p<0.001$ compared in groups by using one-way ANOVA followed by least significant difference post hoc tests. 

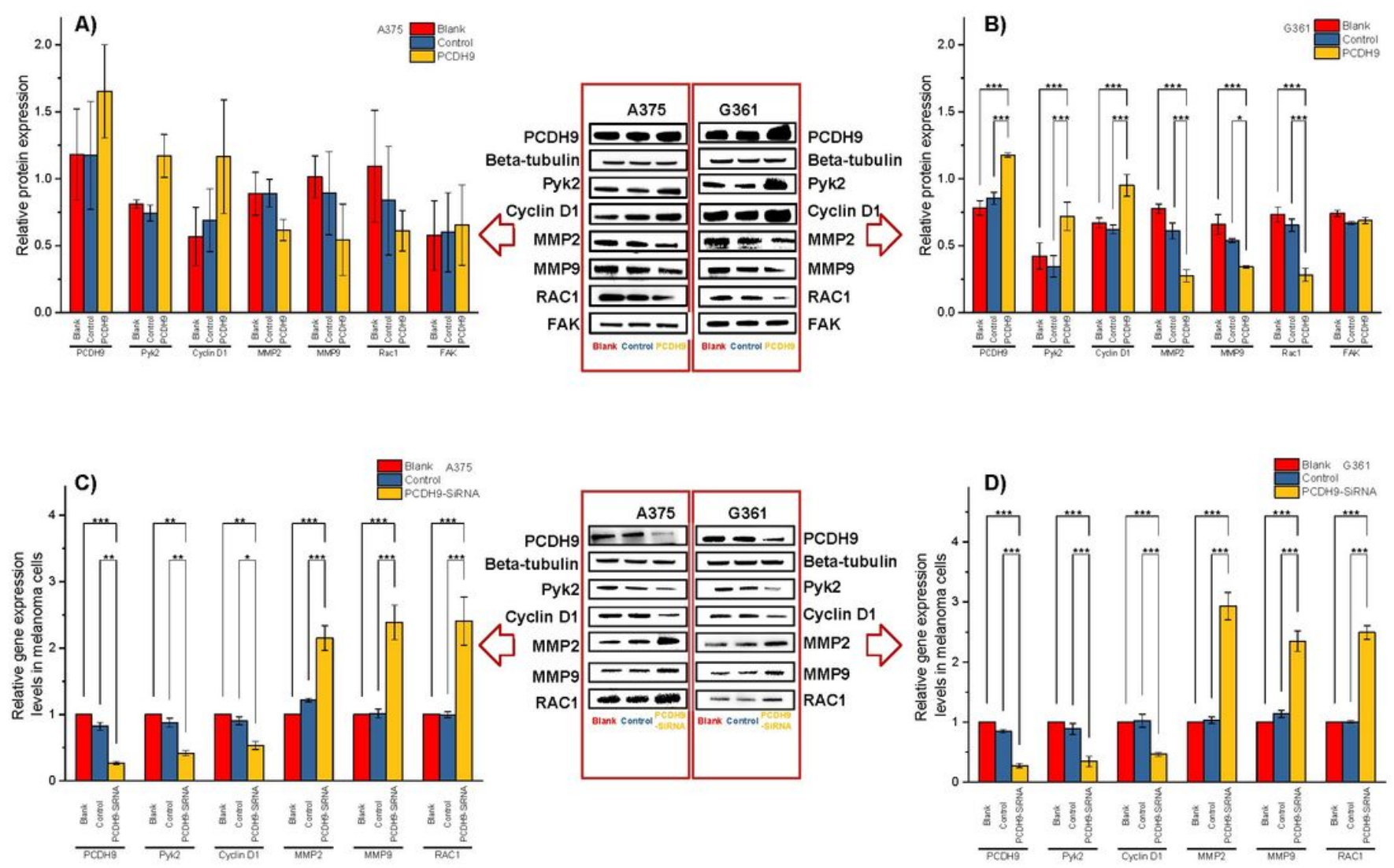

\section{Figure 2}

Effects of overexpressed and interfered PCDH9 in melanoma cells measured by PCR analysis. (A) The expressions of PCDH9 were significantly upregulated by lentivirus infection. Overexpressed PCDH9 significantly upregulated the gene expressions of Pyk2 and Cyclin D1 and downregulated RAC1, MMP1 and MMP9 except FAK in A375 (B) and G361 (C) cells. Interfered PCDH9 downregulated the gene expressions of Pyk2 and Cyclin D1 and upregulated RAC1, MMP1 and MMP9 in A375 (D) and G361 (E) cells. ${ }^{*} p<0.05,{ }^{*} p<0.01,{ }^{* \star *} p<0.001$ compared in groups by using one-way ANOVA followed by least significant difference post hoc tests. 

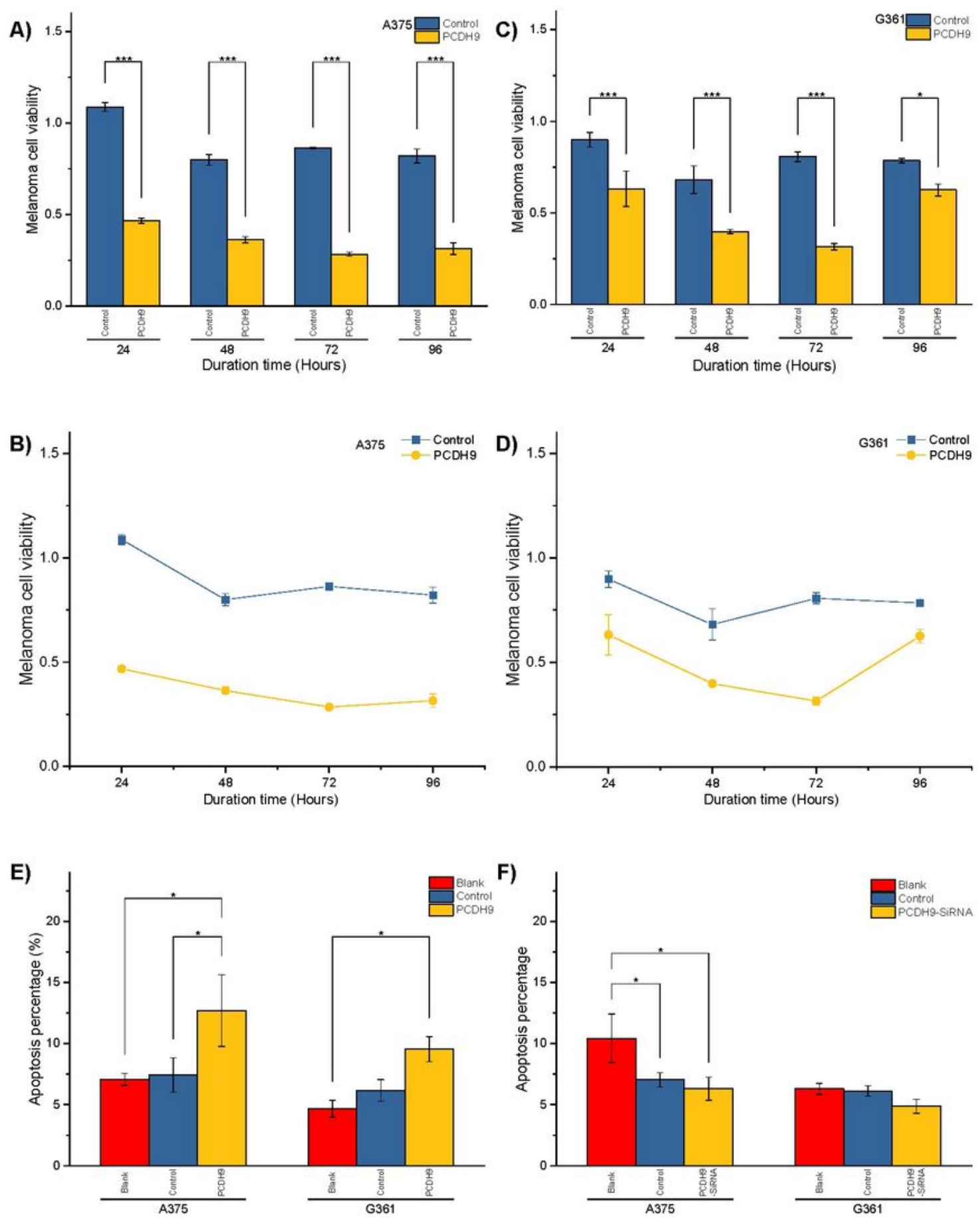

\section{Figure 3}

The viability of melanoma cells significantly was reduced by overexpressed PCDH9 in A375 (A, B) and G361 (C, D) cells. * $p<0.05,{ }^{*} p<0.01,{ }^{* \star *} p<0.001$ compared with control groups by using Student's $t$ tests. The alteration of PCDH9 expression significantly affected the apoptosis of melanoma cells. The overexpression of PCDH9 significantly promoted apoptosis in both cell lines (E), while the interference of PCDH9 reduced apoptosis in A375 cell line and in a more modest manner in G361 cell lines (F). * $p<0.05$, 
$\star \star p<0.01, * \star \star p p<0.001$ compared in groups by using one-way ANOVA followed by least significant difference post hoc tests.
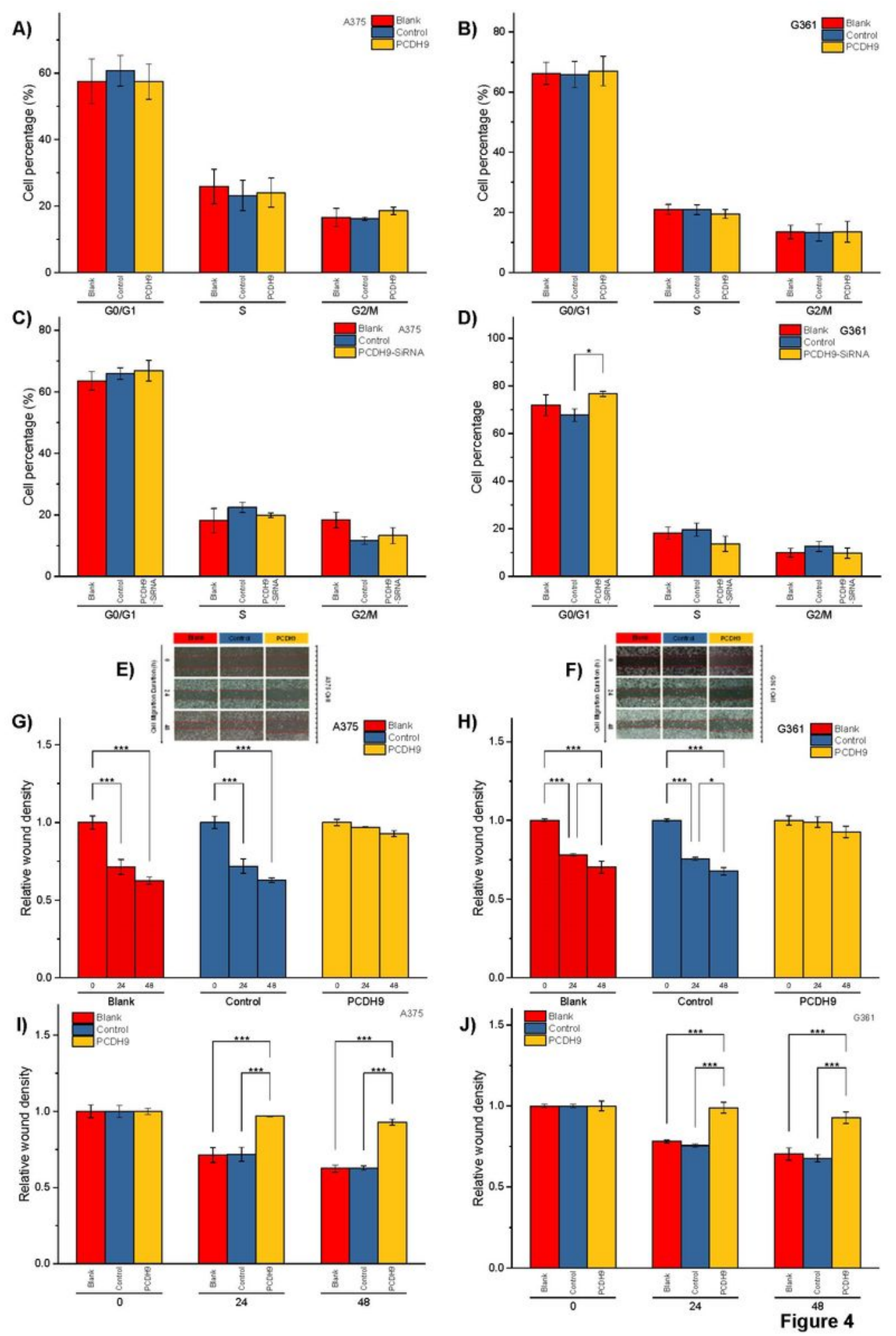

Figure 4

The varieties of PCDH9 expression did not significantly affect melanoma cell regulation. The cell percentage of melanoma cells affected by overexpressed PCDH9 in different cell period time in A375 (A) and G361 (B) cell lines. The cell percentage of melanoma cells in A375 (C) and G361 (D) cell lines. * 
$p<0.05,{ }^{*} p<0.01,{ }^{*} \times \mathrm{p}<0.001$ compared in groups by using one-way ANOVA followed by least significant difference post hoc tests. The scratched boundary of migrator cells was observed by inverted microscope DMI3000B (Leica, Germany). Representative image of melanoma cells invaded from the scratched boundary in A375 (E) and G361 (F) cell lines. Relative density of scratched boundary wasn't altered in overexpressed PCDH9 groups comparing with blank and control groups in A375 (G) and G361 (H) cell lines. Relative density of scratched boundary was significant different in overexpressed PCDH9 groups after 24 and 48 hours in A375 (I) and G361 (J) cell lines. * $p<0.05$, ** $p<0.01$, *** $p<0.001$ compared in groups by using one-way ANOVA followed by least significant difference post hoc tests.
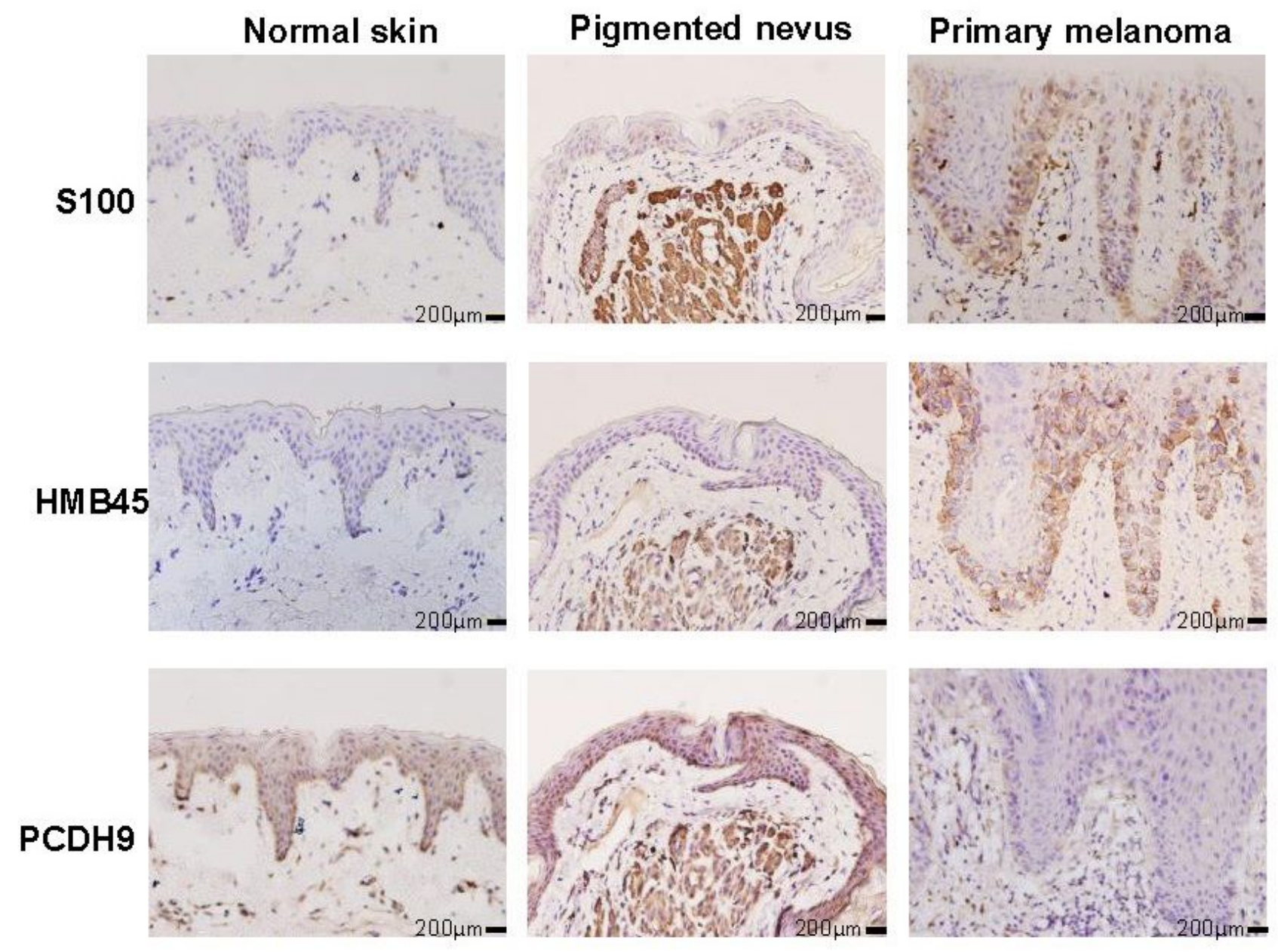

\section{Figure 5}

Immunohistochemical analyses of PCDH9 expression in normal skin, pigmented nevus, and melanoma tissue. Positive percentage of PCDH9 expression was lower in human melanoma tissue than normal skin or/and pigmented nevus tissue. PCDH9 was mainly expressed in cytoplasm, while a small amount in nuclei. S100 and HMB45 are melanoma markers. The scale bar represents $200 \mu \mathrm{m}$. 


\section{Supplementary Files}

This is a list of supplementary files associated with this preprint. Click to download.

- SupplementaryMaterial.pdf

- GraphicAbstract.jpg 